\title{
AvatARes de la BioÉtica en la déCADA del 90 en CUBA
}

\author{
Avatars of Bioethics in the 90's in Cuba
}

RAYDEL ZUMETA FERNÁNDEZ

Universidad de las Ciencias Informáticas, Cuba

\section{KEY WORDS}

Bioethics

Social conditioners

Institutionalization

\begin{abstract}
This paper explains the social factors that condition the appearance of bioethics in Cuba, its ups and downs, its successes and failures, with the purpose of clarifying the stages through which the matter passes and contributing to an understanding of its epistemological status. It is stated that the 1990s marked the formal origin or processes of institutionalization of bioethics in Cuba. It is argued that the new discipline was not welcomed by many government officials. The crisis of the 1990s marked a new route in the social relations of production in Cuba whose most sensitive consequence was the restructuring of the hierarchy of values, with the demise of the socialist camp. The consequences of the surreptitious disappearance of the socialist camp are exposed.
\end{abstract}

PALABRAS CLAVE

Bioética

Condicionantes sociales

Institucionalización

\section{RESUMEN}

En el presente trabajo se explican los factores sociales que condicionan la aparición de la Bioética en Cuba, sus avatares, aciertos y desaciertos, con el propósito de esclarecer las etapas por las que transita la materia y coadyuvar a una comprensión de su estatuto epistemológico. Se expone que los años 90 marcan el origen formal o procesos de institucionalización de la Bioética en Cuba. Se fundamenta que la nueva disciplina no fue acogida con regocijo por muchos funcionarios gubernamentales. La crisis de la década de 1990 momento marcó una nueva ruta en las relaciones sociales de producción en Cuba cuya consecuencia más sensible fue la reestructuración de la jerarquía de valores, al fenecer el campo socialista. Se exponen las consecuencias de la subrepticia desaparición del campo socialista.

Recibido: 03/06/2019

Aceptado: 01/11/2019

\section{GLOBAL俄 KNOWLEDGE




\section{Introducción}

$\mathrm{L}$ a Bioética es una disciplina cuyo origen se debe a la idea de Van Rensselaer Potter, bioquímico, oncólogo e investigador norteamericano quien acuña el término Bioética (bios: vida y ethos: ética), para mostrar la necesidad de reorientar la filosofía práctica de la vida, para profundizar en el significado de la misma y en ella el sentido de la nuestra.

Esta materia pretende humanizar el quehacer de la ciencia y la técnica, a raíz de la maduración del pensamiento contemporáneo propiciada por el auge que adquirió el desarrollo científico y tecnológico en las décadas del 60 y el 70 y los impactos negativos de la misma en la pluralidad geográfica. Su estudio manifiesta una creciente aceptación académica y social; amén de la visión biomédica que inicialmente le estampó la propuesta del Kennedy Institute of Ethics de la Universidad de Georgetown en Washington, la idea originaria de Potter cobra fuerza en los últimos años del pasado siglo y los primeros del XXI. En la actualidad se columbra ese retorno a la idea original de la Bioética propuesta por Potter, sobre todo en las zonas geográficas de la periferia.

En este artículo se asume que es la Bioética una materia que promueve la integración de los valores, el conocimiento y la práctica, que busca contribuir a la supervivencia y al mejoramiento de la condición humana que significan hoy: por un lado, eliminar las agresiones, proteger y conservar la naturaleza, prevenir y enfrentar enfermedades y catástrofes; por otra parte, tiene como fin que las personas puedan adquirir bienes materiales y espirituales necesarios para una vida digna, que exista una relación dialógica entre ellos, que tengan acceso a la educación y que sean protagonistas en la toma de decisiones y en la construcción social.

Los años 90 marcan el origen formal de la Bioética en Cuba, aunque ya se habían desarrollado eventos y divulgadas memorias escritas que abordaban los problemas cuya solución competen a esta materia. Tuvo gran influencia en su aceptación y aprehensión la publicación de un colectivo de autores cubanos acerca de la definición de los criterios para determinar la muerte humana a partir de la pérdida irreversible de las funciones neurofisiológicas que favorecieron un año más tarde la celebración del I Simposio Internacional sobre Muerte Encefálica en septiembre de 1992 en La Habana.

En este evento se dedicó un espacio para el debate de dilemas y/o conflictos de valores que se generan en el sector sanitario. Hasta esa fecha, si bien ya se conocía el vocablo, no se usaba en la isla la palabra Bioética, de hecho, cuando en esa misma fecha se inauguró en el Hospital Hermanos Ameijeiras el primer comité que estaba encaminado a resolver los casos dilemáticos que se presentaran en esa institución de nivel terciario, así como a la educación en Bioética de todo el personal recibió el nombre de Comité de Ética Clínica y no el de Bioética que era lo que realmente se estaba haciendo en estas comisiones.

Sin embargo, a pesar de las substanciales transformaciones que se vivieron en Cuba en el primer lustro de los años 90 de la vigésima centuria, la necesidad de solventar la profunda crisis de la moralidad, sustentadas en profundas carencias económicas, la aprobación que tuvo en algunos espacios, la Bioética no fue acogida con regocijo por muchos funcionarios gubernamentales, quienes la tildaron como un arma ideológica del enemigo; por lo que sus primeros pasos en Cuba fueron dados, sin la validación del poder, por sendas escabrosas y matizados por incomprensiones políticas y la ortodoxia en la academia cubana, más las nuevas prácticas que comienzan a generalizarse en una población que, abrumada por la frustración que la gama de problemas que las insuficiencias materiales acarrean, comenzó de manera ostensible a desatender todo lo ajeno a los problemas económicos.

Para mejor compresión de este transitar plagado de discrepancias hay que destacar el contexto en que se origina y desarrolla en nuestro país, ya que parte de esta historia aun subyace en penumbras amén de haberse institucionalizado ya la enseñanza e investigación de la Bioética, de contar dicha disciplina con un programa de maestría de las cuales han nacido varias tesis, publicaciones diversas, numerosos eventos científicos efectuados.

Siendo ese periodo inicial de la Bioética en Cuba convulso y en gran medida desconocido 
para las nuevas generaciones que estudian esta materia se pretende explicar en este artículo las condicionantes sociales que caracterizaron la eclosión de la Bioética en los años 90, sus avatares, aciertos y desaciertos, en aras de elucidar su origen, así como comprender y acomodar sus bases epistémicas a las características de la realidad cubana.

\section{Diseño metodológico}

\section{Métodos}

- Método histórico: Facilitó la aproximación a los referentes teóricos del tema, así como profundizar en las condicionantes sociales que explican los años iniciales de la Bioética en Cuba.

- Sistematización: Permitió desarrollar el objeto de la investigación mediante la integración de herramientas metodológicas aportadas por tres campos de conocimiento: la Historia, la Bioética y los estudios CTS; favoreció la confección de la periodización de la Bioética en Cuba y el análisis de sus principales condicionante sociales.

- Análisis de contenido: Posibilitó el estudio de la bibliografía concretando aquella de interés para la investigación con el propósito de determinar el sistema de conceptos y categorías. Permitió extraer las relaciones esenciales del objeto investigado para descubrir y estudiar nuevas cualidades, relaciones, principios o leyes del mismo.

\section{Técnicas y procedimientos}

\section{Técnicas de obtención de información y recolección de datos}

- Revisión bibliográfica y documental: Para identificar las premisas teóricas de los temas que se abordan en este estudio, se realizó un análisis de la bibliografía nacional e internacional disponible.

- Se realizó un panel de expertos a fin de elaborar una guía de observación para ser aplicada a especialistas en las temáticas Bioéticas en entrevistas a profundidad y un grupo focal, a fin de conocer sus criterios acerca de los aspectos inherentes al problema de investigación. Se consideraron como expertos a Doctores en Ciencias Filosóficas que en los últimos cinco años hubieran publicado o impartido docencia en temas de Bioética. El panel estuvo integrado por tres expertos (Dr. C José Ramón Acosta Sariego; Dr. C Leonardo B. Pérez Gallardo; Dr. Calixto Machado Curbelo), a los que se les presentó una primera versión de la guía, la cual quedó definitivamente conformada tras realizar el análisis de contenido de las respuestas de dichos expertos

- Las entrevistas a profundidad se realizaron en una sesión, en horario de la tarde y en condiciones de privacidad. Todas las entrevistas fueron guiadas por los autores de la presente investigación.

- La población bajo estudio fueron especialistas con dedicación a las temáticas de Bioética. (10)

\section{Técnicas de análisis y elaboración}

- Agrupación y sistematización de los contenidos de acuerdo al objetivo general. Para integrar los núcleos teóricos de los principales períodos por los que transita la Bioética en Cuba.

- Se realizó un análisis de documentos a partir de los criterios emitidos en las entrevistas a especialistas en las temáticas Bioéticas.

\section{Consideraciones éticas particulares de este estudio}

A todos los especialistas entrevistados se les solicitó su consentimiento informado, explicándoles los objetivos y características de la investigación, y se les aseguró que sus opiniones permanecerían en el anonimato al realizar el informe final de la misma y cualquier otro tipo de socialización de los resultados, cuyo interés sería la tendencia general y no los criterios individuales, cuyos contenidos solo se tomarían a modo de ejemplo, sin revelar la fuente.

\section{Control semántico}

Condicionantes sociales: Situación o circunstancia indispensable para la existencia de otra. 
Periodización: Establecer períodos para un proceso histórico, cultural, científico, etc.

\section{Desarrollo}

En los años 80 y en los inicios de los 90 se produce en Cuba un escenario que demandará de otro tipo de tratamiento desde el punto de vista moral. La ética tradicional, a raíz de la incorporación de nuevas tecnologías, principalmente en el campo de salud, evidencia su incapacidad resolutiva ante los nuevos problemas que acarrean las nuevas herramientas y abre el diapasón a un nuevo discurso que moralmente sostenga los pilares de la sociedad cubana que se sumerge, con la caída del campo socialista, en la bruma de la moral capitalista, y pone en riesgo la vitalidad de su proyecto social. La Bioética sería esa disciplina que llenaría los espacios que la ética tradicional no pudo suplir, y, por tanto, debería constituir la nueva postura ética por excelencia en un contexto como el nuestro dónde el desarrollo pleno del hombre, el amor por la vida en sentido general, el respeto al derecho ajeno, la dignidad, entre otros valores afines ocupan un lugar privilegiado y la corriente neoliberal dificulta la visibilidad de los mismos.

Sobre la necesidad de incorporar el nuevo saber al escenario académico cubano, interesante resulta el planteamiento de Bravo Matarazzo acerca de este punto, no solo por dotar de flexibilidad a la relación médicopaciente, mediante el quebrantamiento del rígido paternalismo médico $\mathrm{y}$ las premisas deontológicas que prevalecen en estas ramas de la salud; o la extensión del sujeto moral a la naturaleza, sino que trasciende a un plano económico donde la interacción con el mercado foráneo implica el conocimiento de sus leyes:

"Si nos referimos al campo de la ética en el terreno biomédico, debemos decir que ello es, además, una necesidad práctica. Por ejemplo, Cuba comparte proyectos biotecnológicos importantes con empresas europeas, las cuales tienen establecidos controles muy estrictos. Si nuestro país aspira a participar del mercado europeo, no tiene otra alternativa que dominar no solo las cuestiones de las patentes, el marketing u otros aspectos, sino debe conocer a la perfección las normas y controles éticos establecidos que forman parte de los contratos.
Por otra parte, cada vez más, las revistas científicas exigen para su publicación que los trabajos acaten las normas vigentes para el trato de animales de laboratorios y ensayos clínicos; lo cual debe ser respetado, porque de otra manera los artículos serían rechazados por los consejos editoriales de esas publicaciones".

El ciclo de conferencias dirigidas por Bravo Matarazzo despertó el entusiasmo por la nueva disciplina en varios autores cubanos. Sin embargo, otros no la asumieron de igual manera, por lo que la institucionalización de la misma fomentó serios debates acerca de la validez de su discurso en nuestro contexto.

Los años 90 marcan el origen formal de la Bioética en Cuba, influyó una publicación de un colectivo de autores cubanos donde se definen los criterios para determinar la muerte humana a partir de la pérdida irreversible de las funciones neurofisiológicas que favorecieron un año más tarde la celebración del I Simposio Internacional sobre Muerte Encefálica en septiembre de 1992 en La Habana.

En este evento fue donde por primera vez se hizo uso del término. Hasta ese momento, si bien existía, no se usaba en la isla el vocablo bioético, de hecho, cuando en esa misma fecha se inauguró en el Hospital Hermanos Ameijeiras el primer comité que estaba encaminado a resolver los casos dilemáticos que se presentaran en esa institución de nivel terciario, así como a la educación en Bioética de todo el personal recibió el nombre de Comité de Ética Clínica y no el de Bioética que era lo que realmente se estaba haciendo en estas comisiones.

Por otro lado, se desarrolló en diciembre de ese propio año 92 la III Jornada Científica del Instituto de Medicina Legal, en el cual se incluyeron estos temas relacionados con la determinación de la muerte humana. Pero no es hasta el año 1994 que comienza su proceso de consolidación en la isla a partir de la creación de un grupo multidisciplinario de profesores quienes promovieron la Bioética en los centros de educación médica superior, compuesto fundamentalmente por docentes de las universidades médicas de La Habana, Santiago de Cuba y Camagüey, los cuales diseñaron un curso intensivo de 40 horas, sustentado en métodos activos de enseñanza y en el debate científico colectivo. 
Sin embargo, la nueva disciplina no fue acogida con regocijo por muchos funcionarios gubernamentales, quienes tildaron a la misma como un arma ideológica del enemigo. Por lo que los primeros pasos de la Bioética en Cuba fueron dados por sendas escabrosas y matizados por incomprensiones políticas y académicas. Para mejor compresión de este transitar plagado de discrepancias hay que destacar el contexto en que se origina y desarrolla en nuestro país.

La isla en esta etapa soporta profundas afectaciones económicas y en consecuencia con esto se produce una reestructuración de la jerarquía de valores, ganando espacio el egoísmo, el individualismo, la corrupción, el oportunismo, entre otras, como formas indispensables para la subsistencia individual. Por su parte, las agresiones procedentes del enemigo del norte obligan a reforzar la defensa de la patria en las diferentes trincheras, fundamentalmente en lo ideológico. Sin embargo, se asumieron posiciones irracionales ante algunos productos provenientes del seno de las sociedades capitalistas.

\section{Condicionantes socio-económicas en la década del 90}

La caída del campo socialista condujo a la pérdida de casi el $70 \%$ de los mercados socialistas, y para hacer más crítica aún la asimetría, el bloqueo se profundizó, lo que marcó una nueva ruta en las relaciones sociales de producción en Cuba. Bajo tales condicionantes la isla entró en serias dificultades económicas que, plantea el profesor universitario Esteban Morales, solo recientemente comienza a superar (Morales, 1996).

Ante la catástrofe económica los líderes de la revolución lanzaron en el año 91 el llamado Período Especial, estrategia que consiste en la instauración de una economía de guerra en tiempos de paz para poder arrostrar la crisis a que nos abocaron los hechos acontecidos en la arena foránea a finales de la década del 80 y principio de los 90.

Desde el exterior se vaticinaba que el desplome de la Revolución cubana era cuestión de días o de semanas. No obstante, se dictaminaron disposiciones dirigidas al logro de la elevación de la eficiencia económica y la competitividad, y la reparación financiera interna; se propusieron, además, soluciones al endeudamiento del país; se promovió la reinserción en la economía capitalista, se incentivó la inversión de capital extranjero, el fortalecimiento de la empresa estatal cubana, condición esta necesaria y sin la cual no puede haber socialismo. También se analizó la necesidad de ampliar y perfeccionar los cambios económicos que fuese necesario hacer, de manera gradual y ordenada.

En 1991 se realizó el IV Congreso del PCC. En este cónclave se examinó la situación del país y se enfatizó en la necesidad de salvar la Patria, la revolución y el Socialismo, haciendo loable justicia a la obra que tanta sangre, sacrificio y esfuerzo había costado al pueblo cubano en más de cien años de lucha. Se tomaron importantes acuerdos en lo tocante a las modificaciones a la Constitución, los estatutos del Partido y se asentaron los cimientos de una planificación orientada al sustento de los pilares ideológicos y comenzar la recuperación. La tenacidad de la revolución cubana provocó un recrudecimiento, por parte de los círculos apátridas de Miami y el gobierno norteamericano, de las campañas difamatorias contra la revolución, la desestabilización ideológica y un sensible incremento del bloqueo económico.

Las estrategias económicas trazadas por el gobierno revolucionario lograron preservar la continuidad de nuestro proceso, pero se notaron cambios apreciables en el imaginario cotidiano de la sociedad, como consecuencia de las privaciones materiales y la necesidad de sobrevivir, aupada por la reestructuración de la jerarquía de valores, la irreversible invasión cultural y la desazón espiritual latente. Varias de las medidas tomadas para salvar la revolución tuvieron resultados que desestabilizaron el modelo económico, que hasta ese momento había predominado. La economía planificada y todos los beneficios que de ella se derivan entraron en peligro de extinción cuando se autoriza la creación de empresas mixtas con capital extranjero, además se permite en el año 1992 a las empresas con $100 \%$ de capital nacional a que puedan operar en dólares. 
En 1993 se oficializa la doble circulación monetaria, coexisten de esta forma el peso y el dólar. Se fomenta la autonomía empresarial y se descentraliza el comercio exterior. Si se añade a estos factores la creciente falta de control efectivo por parte de los trabajadores y la existencia de una burocracia que no está sujeta a ningún control político por parte de la población, se tendrá como resultado un efecto corrosivo en la moral, en la planificación económica y acelera el proceso de diferenciación social, echando agua en el molino de la contrarrevolución capitalista. La doble circulación agudiza los desequilibrios sociales y económicos, fortalecido tal lastre por la gran diferencia que hay entre el cambio oficial y el del mercado negro. El desarrollo del turismo en el país constituyó un riesgo para nuestro pueblo; drogas, pornografía, prostitución y otros lastres sociales sin precedentes en la Cuba revolucionaria.

El nivel de vida en el país disminuyó durante los primeros años de los 90 considerablemente, pues se redujo en casi el $50 \%$ lo cual condujo, entre otras condicionantes sociales, a que muchas personas se trasladaran hacia centros laborales mejor remunerados, principalmente relacionados con el turismo lo cual ha redundado en un empeoramiento constante de los demás servicios y en la productividad del trabajo, y que aparezcan violaciones de magnitudes que prácticamente desaparecidas de nuestro entramado por el bienestar social experimentado en las décadas del 70 y el 80. Las carencias materiales afectaron sectores como el de la salud, aunque en esta rama no se percibe ese traslado masivo hacia otros campos como sí sucedió en el terreno de la educación.

Sin embargo, Cuba siguió siendo, gracias a los encomiables esfuerzos del gobierno, una potencia en la educación y la salud. El líder histórico, Fidel Castro, en su discurso de clausura del IV Congreso del PCC reafirmó la continuidad de nuestro proceso revolucionario en varias aristas: "Haremos lo que haya que hacer; trabajaremos lo que haya que trabajar; resolveremos los problemas que esté en nuestras manos resolver; continuaremos en cualquier circunstancia nuestros programas, en primer lugar el programa alimentario, aunque falten piensos y falten fertilizantes; continuaremos desarrollando nuestro programa de biotecnología, industria farmacéutica y equipos médicos" (Castro, 1991).

La segunda mitad de la década del noventa, por ejemplo, mostró el crecimiento en términos económicos de la isla. Precisamente en el año 1994 se expresa la reversión de este decaimiento cuando se muestra que la tasa de crecimiento promedio anual es de $4,7 \%$, superior a la del $3 \%$ experimentada por la región latinoamericana. Se nota una mayor independencia económica con respecto a épocas anteriores donde estuvimos sujetos a las tendencias del mercado español, norteamericano o el soviético, comienzan a desarrollarse las relaciones económicas con los países de América Latina y el Caribe, en esencia indica este año la afiliación de Cuba a un mercado al que no había estado integrada desde 1960.

El impacto de la crisis económica de los primeros años de la década del 90 repercute y de manera profunda en los referentes espirituales. Las soluciones para solventar las fisuras económicas no trascendieron este enfoque; en el aspecto moral se mostraron profundos cambios. Las limitaciones de la ética tradicional para solucionar situaciones nacidas en las últimas décadas del siglo XX y acentuadas en los umbrales de XXI y su impacto en Cuba, los importantes avances que se columbran en la ciencia y la tecnología resultantes de las políticas lanzadas por el gobierno desde sus primeros años en el poder, la maduración de la sociedad cubana como consecuencia la crisis de los 90, la crisis de valores y la perentoria invasión cultural del imperialismo mundial, entre otros factores, animan la aparición de nuevas perspectivas académicas que reconstruyan la idea del bien en un país donde las fronteras de lo bueno y lo malo se han fundido en el fragor de la escasez, un concepto sustentado en posiciones holísticas, integradora y unitivas, que incluyan el sentir de la pluralidad contextual, generacional, de género y racial, que retome ese ideal humanista retornando al legado histórico de sus próceres.

Amén de las alertas que suscitaron las sacudidas en la sociedad, muchos de los decisores de las políticas públicas no tuvieron la lumbrera suficiente para avizorar en la Bioética los elementos para sustentar con un conveniente 
marco axiológico los rumbos nuevos que ahora enfrentaba la revolución cubana.

\section{Las reticencias del poder en el desarrollo de la Bioética en la década del 90}

A decir de muchos bioeticistas cubanos las condicionantes políticas de los años 90 signaron el devenir de la Bioética en la isla. Los conflictos que se dieron en este período, las agresiones y desmanes que sufrieron los partidarios del nuevo modo de reflexión no son reseñados en la literatura, amén de que se ha hecho mención en algunos textos, lo difíciles que fueron estos primeros cinco años para el desarrollo de la Bioética en Cuba.

Muchos de los entrevistados plantean la hostilidad y el escepticismo por parte de algunos funcionarios hacia el nuevo saber. Sin embargo, sus respuestas no explicitan los hechos negativos individuales que tuvieron que arrostrar, los eventos que cerraron sus puertas a los temas Bioéticas, las instituciones que miraron de reojo la asimilación de la nueva disciplina, la señalización de las personas que en nombre de principios inexistentes tanto desde lo jurídico, lo histórico como lo ético, el temor de desviaciones ideológicas y el desconocimiento de qué de la Bioética sirvieron de barrera al debate bioético en el país. La bibliografía consultada tampoco muestra el impacto del factor político en el recorrido de la nueva disciplina, lo cual obliga al estudio de la conformación de ese pensamiento en el período posterior al 59 para comprender la influencia de esta condicionante social en la trayectoria de la Bioética. Es fundamental el desarrollo de este punto

En 1961 se crea la Escuela de Ciencias Políticas, la cual perteneció a la Facultad de Humanidades de la Universidad de la Habana, y cuya dirección recayó en los Profesores Raúl Roa García y Pelegrín Torras de la Luz. El propósito de esta institución fue, inicialmente, formar futuros diplomáticos y periodistas y tuvo un influjo decisivo en la formación de la actual generación de politólogos cubanos. Estos centros, además estuvieron se caracterizaron por el sesgo marxista que se le imprimió a toda la política educacional y cultural de la Revolución en aquellos años fundacionales y los años que le precedieron.

Sin embargo, en dicha institución y de manera particular en las publicaciones del titulado órgano oficioso del Departamento de Filosofía de la Universidad de la Habana Pensamiento Crítico, primaba el apotegma expuesto por el Profesor Roa en su obra Historia de las Doctrinas Sociales:

En la historia de las doctrinas sociales hay que penetrar con el ademán sereno y la pupila limpia de prejuicios y su exposición académica debe estar presidida por la más pulcra objetividad. En ningún terreno, como en el de nuestra ciencia, son tan múltiples y variados los criterios, las perspectivas y las soluciones propuestas. Cada estudiante queda, por consiguiente, en libertad absoluta de adoptar el que estime por conveniente o de no adoptar ninguno. $\mathrm{Ni}$ se propone ni se impone: se expone. El espíritu científico y la intolerancia son incompatibles. El espíritu científico se nutre y enraíza en la libertad de investigación y crítica. La intolerancia -"esa extensión hacia fuera del dominio exclusivo ejercido dentro de nosotros por la fe dogmática"- intoxica la inteligencia, deforma la sensibilidad y frustra la actividad científica, que es impulso libérrimo hacia la conquista y posesión de la libertad. (Alzugaray, 2005)

Al respecto expone Alzuragay que en la labor ensayística y en sus discursos políticos, los principales dirigentes de la Revolución, principalmente nuestro líder histórico Fidel Castro, Ernesto "Ché" Guevara y Carlos Rafael Rodríguez, promovieron una visión del marxismo alejada de todo esquematismo y dogmatismo. En este período el pensamiento del Che ejerce gran influencia en los jóvenes politólogos cuyas ideas gravitaban alrededor de la revista Pensamiento Crítico y que dejó de ser publicada en una etapa que muchos de nuestros intelectuales han denominado el quinquenio gris, provocado por la contaminación de nuestra ideología por los códigos del realismo socialista proveniente de la extinta Unión Soviética.

Las dos décadas siguientes, o sea, la del 70 y la del 80, fueron escenario de la agudización de esta situación y por tanto inhibió el desarrollo de las ciencias políticas cubanas. Desparece precisamente en este decenio la Escuela de Ciencias Políticas y parte de sus funciones fueron 
asumidas por la Escuela Superior del Partido Comunista de Cuba Antonio "Ñico" López la cual tendría como objetivo central la formación de cuadros de esta organización y expedía y expide, al menos hasta el 2005, un título de Licenciado en Ciencias Sociales. Los politólogos que en ella trabajaban, dirigidos por su Rector, Raúl Valdés Vivó, se caracterizan por su apego a los clásicos del marxismo Marx, Engels y Lenin.

Bajo la influencia del dogmatismo que emanaba de los centros de estudio e investigación de la antigua Unión Soviética, se modificaron programas de estudio y desaparecieron algunas de las publicaciones señaladas, aunque otras, como la Revista Casa, mantuvieron el vínculo con el pensamiento político latinoamericano y caribeño. Para las ciencias sociales soviéticas, el materialismo histórico sustituía no sólo a la ciencia política, sino también a la sociología. Por tanto, los estudios de la política se basaron en los manuales soviéticos y eran asumidos dentro del programa de Filosofía marxista II, obligatorio para todas las carreras universitarias, la primera parte se dedicaba al materialismo dialéctico.

Estos elementos favorecieron que la inflexibilidad ganara terreno, tanto en la conceptualización teórica como en el método de análisis y que en los años posteriores el desarrollo de las ideas políticas en Cuba fueran tildadas por más de uno como ortodoxas y rígidas, amén de que muchas personalidades se autonombraran a sí mismas marxistas sin examinar la desunión existente entre su declaración de partidismo filosófico y el desconocimiento, subestimación o no utilización del método de análisis del marxismo, el materialismo dialéctico e histórico. La destacada bioeticista cubana María del Carmen Amaro Cano al respecto recordó la advertencia de Gramsci a los marxistas de su época: "Marx no ha escrito un credillo, no es un mesías que hubiera dejado una ristra de parábolas cargadas de imperativos categóricos, de normas indiscutibles, absolutas, fuera de las categorías del tiempo y del espacio" (Cano, 2008).

Afirma Amaro Cano que, en Cuba, tal como sobrevino en otros lugares, los dogmáticos apoyados en la ortodoxia petrificaron la teoría del marxismo obviando la esencia de las herramientas metodológicas que esta aporta. El primer lustro de quehacer bioético en Cuba estuvo signado por la rigidez y la censura hacia la disciplina ante el temor que entraña la penetración ideológica implícita en la política norteamericana hacia la isla. La incomprensión, la crítica irracional a lo diferente, el aislamiento de temas por los conflictos que emanan de sus análisis restaron fuerza moral y credibilidad a los principios metodológicos marxistas.

Apunta Ambrosio Fornet lo siguiente:

A la Bioética en Cuba se le acusó, desde esas posiciones ortodoxas, de ser arma del enemigo dado su lugar de procedencia, su validez fue cuestionada aduciendo que es un genuino producto norteamericano, cuya aplicación es efectiva para el contexto de esa sociedad neoliberal, caracterizada por un desmedido crecimiento económico a expensas del desarrollo humano; donde la exaltación del principio de la autonomía, sustentado en las bases de la filosofía neoliberal, centraliza su atención en la libertad individual desconociendo los intereses o derechos sociales en general. Estos criterios mostraron que no hubo en un inicio un acercamiento científico al tema por parte de sus detractores. (Amaro, 2008)

La incorporación de la Bioética como reajuste teórico y cosmovisivo resultante de los nuevos problemas o conflictos de valores que arrostran los cubanos en los años 90 es de vital importancia ya que su propuesta trasciende los servicios sanitarios para adentrarse en otros aspectos de la vida social. En aquellos momentos de transición económica de la sociedad cubana, con la introducción de inversiones extranjeras y otros factores de conflicto que a priori ponían en peligro una escala de valores establecidos durante el proceso revolucionario, muchas personalidades del país alertaron sobre la necesidad de privilegiar las cuestiones de la ética.

Amén de las posiciones de la ortodoxia el triunfo de la flexibilidad se hizo patente gracias al esfuerzo y la visión de futuro de quienes defendieron la asimilación de la Bioética, en un primer momento como solución a los conflictos o problemas de valores de nuevo tipo que tienen lugar en los servicios sanitarios. A mediados de 
la década del 90 surgen en la isla varias instituciones que serán expresión del grado creciente de comprensión y aceptación del estatuto epistemológico de la nueva disciplina. Hay que destacar que la ciencia política cubana en ese momento comienza a brindar los frutos de la estrategia trazada a inicios de la década con el propósito de rebasar el estancamiento a que estuvo abocado dicha materia.

Se reinician las publicaciones periódicas dedicadas, total o parcialmente, al análisis politológico. Indica Alzuragay que comienzan a publicarse nuevamente las revistas Temas, Contracorriente, y Marx Ahora además del restablecimiento de otras dos revistas importantes que habían dejado de publicarse: Cuba Socialista (órgano teórico del CC del Partido Comunista de Cuba) y la Revista de Política Internacional del ISRI. Estas publicaciones, conjuntamente con otras que no se mencionan, favorecieron la divulgación de las obras de politólogos y sociólogos como Rafael Hernández, Enrique Ubieta, Esteban Morales, Isabel Jaramillo, Jorge Hernández, Mayra Espina, Aurelio Alonso, Fernando Martínez, Roberto González, Carlos Alzugaray, Juan Valdés Paz, Santiago Pérez, Eduardo Perera, Clara Pulido, David González, Thalía Fung, María Elena Álvarez, José Luis Acanda, Luis Suárez, Isabel Monal, Soraya Castro y otros. A pesar de la variedad de temas que incluyeron estas publicaciones no aparece, al menos en sus inicios artículos referidos a este nuevo saber.

\section{Institucionalización de la Bioética en Cuba}

El Primer Simposio Internacional sobre Muerte Encefálica influyó notablemente en la introducción en las academias de la nueva reflexión. "En este congreso -posteriormente renombrados como de Muerte y Coma-surgen las ideas que tiempo después concretaron los dos primeros cursos internacionales efectuados en nuestro país titulados La universidad médica ante los problemas bioéticos actuales celebrado en octubre de 1993 en la sede del entonces Centro Nacional de Perfeccionamiento Médico y cuyas actividades docentes fueron compartidas por profesores cubanos y norteamericanos (Daniel
Wikler, Stuart Yougner, Alta Charo, William Rudick y Steven Miles entre ellos), e Introducción a la Bioética ofrecido en julio de 1994 en la sede de la entonces Facultad de Salud Pública, por Juan Carlos Tealdi en representación de la Escuela Latinoamericana de Bioética con sede en La Plata, Argentina" (Acosta, 2009).

En el terreno jurídico la inserción de la Bioética fue relativamente rápida, los cambios que se gestaronn principalmente en el plano biomédico abogaron por nuevos y eficaces derechos y deberes para las partes, la calidad y los avances de los servicios médicos teniendo en cuenta las necesidades económicas que del país implicaban una mayor responsabilidad. Es en este espacio de la responsabilidad profesional, señala Nora Cedeño, donde precisamente participa el Derecho, quien con su obligatoriedad y coactividad inherentes, regula los límites de los procederes médicos, y la correspondiente imposición de consecuencias jurídicas (Cedeño, 2008).

En la institucionalización de la Bioética sobresalió la labor de los profesores del Departamento de Derecho Civil y de Familia de la Facultad de Derecho de la Universidad de La Habana quienes en los primeros años de la década del 90 realizaron encuentros sistemáticos para discutir temas de Bioética y Derecho. Asistieron personalidades de renombre internacional en este campo como Francisco León Correa, quien en el año 1993 realizó su primera visita a nuestro país.

Las instituciones, especialmente sanitarias, en este período realizaron una notable labor. A mediados de la década de 1990 comenzaron a fundarse espontáneamente Comités Institucionales de Ética de la Investigación (CEI) en importantes unidades de salud e investigación aunque su validación definitiva estuvo dada cuando en 1997, por Resolución Ministerial no. 110 , se facultó a los directores de unidades del Ministerio de Salud Pública (MINSAP) a crear comités institucionales de ética de la investigación científica, y posteriormente el viceministro, doctor José Jardines dispuso orientar la creación de un Comité de Ética de la Investigación Científica (CEI). Estos últimos órganos tienen la ventaja de su permanencia y por tanto de mayores posibilidades de 
seguimiento de los proyectos previamente aprobados en aquellos centros que lo requieran, para que oficie como órgano asesor o consultivo colegiado, vele por el rigor ético de los proyectos y la ulterior ejecución de las investigaciones (Fleites, Soriano, Torres, 1999). Sin embargo, el 13 de febrero de 1995 se crea el Comité de Ética de la Investigación en el Instituto Nacional de Oncología y Radiobiología (INOR), la temprana conformación de este Comité estuvo propiciada por la categorización del INOR en 1986 como centro de referencia para la práctica e investigación en oncología en Cuba.

Entre los acontecimientos que tuvieron mayor connotación en las aspiraciones de desarrollar la educación Bioética en Cuba resaltó la creación de Cátedras en todo el país en centros médicos a partir del año 94. Ante la carencia de preparación teórica de los docentes debían propiciarse espacios de reflexión y debate acerca de las cuestiones morales más importantes relacionadas con la práctica cotidiana de las profesiones de la salud. Afirma la Dra. Eva Miralles Aguilera que las Cátedras de Bioética tienen una gran responsabilidad y un papel protagónico (Amaro, 2007). En la sesión de apertura de ese I Taller Nacional de Educación en Bioética María del Carmen Amaro, en condición de Presidenta de la Cátedra Coordinadora Nacional de la Disciplina, al referirse a las expectativas de trabajo de las Cátedras, enfatizó en que estaban circunscritas a tres cuestiones fundamentales:

- Lograr una significativa contribución al establecimiento de criterios comunes sobre los aspectos éticos y bioéticos que deberían ser incluidos en la educación de pre-grado, en las distintas carreras.

- Posibilitar la elaboración de un nuevo Programa de Formación Básica en Bioética, para la educación continua.

- Facilitar el desarrollo de grupos de trabajo que se encargaran de diseñar proyectos de investigación que respondieran a los principales problemas actuales de nuestras instituciones de salud (Amaro, 2007).

En el caso particular de la Cátedra fundada en la facultad Gral. Calixto García fueron trazadas las siguientes acciones:
- Propiciar el estrechamiento de relaciones de todos los profesionales pertenecientes a las distintas Cátedras del país.

- Favorecer la acción educativa e investigativa conjunta con las Comisiones de Ética Médica de todas las instituciones del país, con especial énfasis en la Atención Primaria de Salud.

- Privilegiar las investigaciones en sistemas y servicios de salud que trataran los problemas existentes en las relaciones APSAtención Secundaria, para contribuir a la elección de las estrategias más adecuadas para un cambio favorecedor de la calidad continúa de la atención en ambos niveles (Amaro, 2007).

La creación de Cátedras de este tipo benefició la maduración del pensamiento bioético en la isla desde el punto de vista académico e institucional, fomentó la celebración de eventos nacionales e internacionales, propició la publicación de artículos científicos y extranjeros. Estos centros no contaron con respaldo jurídico en un inicio, se fueron fundando en la medida que el momento demandaba de su presencia. Aun para esta fecha eran candentes los debates acerca del origen de la Bioética, de ahí que surgió la necesidad de validar jurídicamente estos centros y por tal razón el Viceministro a cargo de la Docencia del MINSAP decidió dictar la Instrucción VAD 5/95, sobre las Cátedras Honoríficas y Multidisciplinarias que reconoció explícitamente la existencia de estos órganos docentes y su papel en el subsistema de la Educación Médica (Acosta, 2009).

En medio de la proliferación de Cátedras de Bioética en el país se celebró en noviembre de 1995 el Seminario Taller Bioética e investigación. Acudieron a este encuentro 84 delegados e invitados procedentes de 14 naciones, así como 258 observadores de diversas instituciones cubanas científicas y académicas (Hodelín, 2011). Dicho evento estuvo dedicado al tema de Ética de la Investigación Científica y devino en un escenario conveniente para confrontar al pensamiento bioético cubano con los derroteros que había seguido en nuestra región. Según Acosta, tal acontecimiento fue el primero de carácter internacional realizado en nuestro país dedicado explícitamente a la Bioética (Acosta, 
2009). Las palabras de clausura estuvieron a cargo de Dr. Julio Montt Momberg, en aquel entonces Director del Programa Regional de Bioética para América Latina y el Caribe. Reseña Ricardo Hodelín un fragmento de la conferencia pronunciada por el Dr. Julio Montt Momberg y que ha servido de faro al quehacer de los bioeticistas cubanos: “... el Programa continuará siendo un lugar de diálogo de todas las disciplinas de la salud en un sentido ampliamente humanista, neutral y respetuoso de todas las opiniones" (Montt, 1995).

En febrero del año 96 el bioeticista español Javier Gafo impartió un curso de postgrado en la Universidad de La Habana titulado Reproducción asistida e ingeniería genética, en estas conferencias participaron 40 cursistas, fundamentalmente dedicados a temas jurídicos. Es válido recordar que la crisis de los años 90 afectó el desarrollo de las Técnicas de Reproducción Asistida ya que los escasos recursos con los que se disponía fueron asignados a la Atención Primaria de Salud y los Servicios de Urgencia, de hecho, la Fertilización in Vitro dejó de ofrecerse durante años (Acosta, 2008). En este sentido cabe señalar que el consenso cubano, coincidió en gran medida con la lógica implícita en la forma en que Javier Gafo expuso el problema, es decir, "no niega la licitud moral del uso de estas técnicas en tanto la infertilidad puede ser asumida como discapacidad y fuente de sufrimiento para la pareja, así como la búsqueda de ayuda médica como constitutiva de acto de amor conyugal, pero evitar las inequidades en el acceso a las mismas y supeditar su empleo a los intereses de bien común" (Acosta, 2008). Tales acontecimientos acercaron a la visión jurídica a los temas bioéticos y la participación activa en la creación y análisis de leyes a partir de los problemas de nuevo tipo que acarrean los adelantos científicos y tecnológicos

En 1997 se creó el Centro de Bioética Juan Pablo II, miembro fundador de la Federación Internacional de Bioética Personalista (FIBIP), bajo la dirección del profesor René Zamora Marín. Esta institución, en coordinación con la Universidad Católica de Valencia, además de haber fomentado la formación de másteres en la disciplina, y la cual ha aportado más de 50 egresados hasta el momento, desarrolló el Primer Congreso Nacional de Bioética en 2003. Entre sus publicaciones sistemáticas se encuentran la Revista Bioética, los Cuadernos del Centro de Bioética Juan Pablo II (selección de artículos) y Reflexiones (monografías); entre sus estudios también sobresalen los cursos de Bioética a distancia, las jornadas anuales de Bioética, el equipo de reflexión Bioética y los cursos de verano.

Se consolidó la Bioética en las academias en esta etapa con la fundación de nuevas instituciones que incluían esta disciplina en el objeto de su misión. Sobresalieron el Centro de Estudios Humanísticos del Instituto Superior de Ciencias Médicas de La Habana (1998) bajo la dirección de María del Carmen Amaro Cano y que se ha destacado por la celebración de cursos, investigaciones y actividades científicas sistemáticas entre las que se cuentan dos talleres nacionales dedicados al tema de la educación en Bioética y varias reuniones nacionales sobre otros temas.

Los principales escritos de bioeticistas cubanos y autores que abordaron este tema fueron publicados en la obra Bioética. Desde una perspectiva cubana, cuyo editor científico fue José R. Acosta. Los textos recogidos en este libro dejan entrever el punto de inflexión hacia la búsqueda del equilibrio necesario entre Bioética médica y Bioética global. Bioética. Desde una perspectiva cubana constituye la cumbre del quehacer literario referido a temas bioéticos, la variedad de temas que se agrupan en esta primera edición van a armar teóricamente a finales de la década de 1990 a los bioeticistas de la isla, lo que allanará el terreno para la incorporación de la Bioética global en el debate acerca del estatuto epistemológico de la disciplina

En ella se recogen más de cuarenta textos de autores cubanos los cuales reflejan, según Acosta, "la continuidad del pensamiento nacional en torno a las cuestiones éticas relacionadas con la salud y la vida que se ha desarrollado en torno al proyecto humanístico de la Revolución, y a la vez marca una ruptura con el modelo clásico hegemónico médico desde la óptica de nuestra tradición de pensamiento que no exalta la individualidad como ha sido típico y tópico en la 
literatura Bioética anglosajona, sino que reconoce el papel del individuo en tanto participa de la responsabilidad colectiva con el bienestar de todos" (Acosta, 2009).

Si bien hasta el momento prevalecía el análisis de los conflictos de valores morales en el campo de una Bioética médica con el rasgo distintivo que le confería el contexto de una salud pública socializada, igualitaria y maximalista las dos primeras ediciones de esta obra incluirían en sus páginas textos significativos que marcarían la tendencia hacia la Bioética global. De lo reseñado por Acosta en su obra, considero meritorio destacar como muestra de esos primeros visos de Bioética global lo planteado por Ubaldo González Pérez, Jorge Grau Avalo y María Antonia Amarillo Mendoza:

...si en Bioética se reconoce y se trabaja encomiásticamente en relación con la calidad de vida en el momento de la muerte...Se debe priorizar la polémica a todos los problemas globales, sociales, grupales e individuales que afectan la vida, el ecosistema y la calidad de vida del género humano, $\mathrm{y}$ también focalizar sobre qué condiciones socioeconómicas de vida, qué garantías jurídicas y qué educación moral necesita el hombre para que sus decisiones no constituyan una violación de los principios morales de la cultura universal. (González, Grau, Amarillo, 1997)

Posterior a la publicación de este texto comienzan a salir a la luz los escritos en los que confluyen el pensamiento ambientalista $y$ bioético cubano, dando comienzo de esta manera a la visión propia de la Bioética global sustentable. Las obras que marcan el giro hacia esta tendencia son, según Acosta: "Ecología y sociedad. Estudios bajo la edición científica de Carlos Jesús Delgado Díaz y Thalía M. Fung Riverón, y Cuba verde. En busca de un modelo para la sustentabilidad también con la edición científica de Carlos J. Delgado" (Acosta, 2009). En estos libros muchos de los planteamientos proponen una ampliación del horizonte bioético:

...Realmente poco haremos si nos ocupamos de los problemas particulares de la salud y no los enfrentamos en su integralidad, en la compleja urdimbre de sus relaciones con los procesos económicos, sociales y culturales del desarrollo... (Acosta, 1999)
El enfoque de la Bioética global incluye en su análisis los problemas del desarrollo y las relaciones económicas y sociales que subyacen en las aplicaciones tecnológicas a la vida social, lo cual descubre la incapacidad del marco teóricometodológico que ha funcionado en el ámbito de la Bioética médica para afrontar los nuevos problemas. Las visiones de estos autores indicaron el camino hacia un sistema categorial que esté compuesto de una mayor solidez teórica para la solución de problemas que rebasan los constreñidos límites de las relaciones interpersonales para penetrar en procesos sociales de carácter histórico, el vínculo del hombre con la naturaleza y el ambiente.

Pero la paulatina asimilación de la Bioética global en Cuba va a estar signada, no solo por la publicación de importantes textos bioéticos que abren el diapasón epistemológico de la disciplina, sino también por el desarrollo de eventos de trascendental magnitud como fueron: el Primer Taller Nacional de Organismos Modificados Genéticamente que se efectuó en el Centro de Ingeniería Genética y Biotecnología en el propio 1999, y un año más tarde, esta misma sede fue el escenario del encuentro de expertos de la UNESCO donde se trató el tema del patrimonio genético y biotecnologías ${ }^{1}$.

Además, el creciente progreso en ramas como la biotecnología demandó una mayor educación e instrucción social. La revista de Biotecnología aplicada fue, desde su primer número publicado en 1984, muy prolija en la presentación de los resultados de las investigaciones que se llevan a cabo en nuestras instituciones. En los últimos años de la década del noventa la misma evidenció una mayor divulgación de los temas bioéticos.

\section{Conclusiones}

La incomprensión que sufrió la Bioética restó velocidad al proceso de institucionalización de la misma. No obstante, la caída del campo socialista, la intensificación de la agresividad del imperialismo y su injusto bloqueo económico, las consecuencias de la globalización neoliberal a partir de los años 90 vista de frente por los

${ }^{1}$ Véase la revista Biotecnología aplicada 16 (número Especial de junio de 1999) y 17(2) de 2000. Disponible en http://elfosscientiae.cigb.edu.cu/ContenidoIssue.asp?ID=90 
cubanos, la agudización de la crisis económica en el país, y el resquebrajamiento de su sistema de valores morales, por un lado, y la pujanza de varios intelectuales comprometidos con el desarrollo de la disciplina, en su mayoría trabajadores del sector de la salud, por otro, abonaron el espacio para el debate y la reflexión bioético, el cual se comenzó a hacer respondiendo a necesidades sentidas y sin definir desde un inicio una base epistemológica acorde a las características de nuestro país.

En la actualidad el pensamiento bioético realizado en Cuba vislumbra ciertas vacuidades teóricas y prácticas (la medicalización de la nueva ética, su concepción de ética aplicada que solapa su holismo), por un lado, mientras que, por otro, muestra una gran originalidad $y$ contextualización del estatuto epistemológico en consonancia con nuestro cuadro axiológico. Su agitada evolución más allá de debilitarla, solidifica su existencia; de sus enfoques caducos emergen otros más flamantes, revolucionarios, necesarios.

El influjo de la academia jugó un papel fundamental en la evolución de la bioética. El resultado de su trabajo está expresado en la numerosa bibliografía que hay acerca de los temas bioéticos, los eventos realizados, las diferentes ediciones de maestrías que se han hecho. Y esto propició que, en el nuevo milenio, ya el debate no fuese acerca de la validez de la bioética para nuestro contexto, sino que el centro del debate de la misma versase acerca de la confección de su estatuto epistemológico. Sobre este punto se puede concluir que a pesar de que la mayoría de los especialistas consultados para este estudio coinciden en que es la bioética una ética aplicada, autores de mucha importancia en los temas referidos a la misma en el país asumen que es esta materia un nuevo tipo de saber y que es hacia esa tendencia que se orienta la recreación del estatuto epistemológico de la bioética en Cuba. 


\section{Referencias}

Acosta J. R. (1999). Bioética, civilización y desarrollo sostenible. En J. Delgado, Cuba verde. En busca de un modelo para la sustentabilidad. La Habana: Editorial José Martí.

- (1999). Bioética. Desde una perspectiva cubana. La Habana: Publicaciones Acuario.

- (2008) Entre el dogma y la razón. El puente bioético de Javier Gafo. Revista Latinoamericana de Bioética. Disponible en http://www.umng.edu.co/www/resources/rbioetica1.pdf. Consultado 22/11/2018.

- (2009). Los árboles y el bosque. Texto y contexto bioético cubano. La Habana: Editorial Félix Valera.

Alzuragay Treto, C. (2005). La ciencia política en Cuba: del estancamiento a la renovación (1980-2005). Revista Ciencia Política, 25(1). Disponible en http://www.scielo.cl/pdf/revcipol/v25n1/art10.pdf. Consultado 23/11/2017.

Amaro Cano, M. del C. (2007). Informe Central al II Taller Nacional de Educación en Bioética. Instituto Superior de Ciencias. Revista Habanera de Ciencias Médicas, 6(5). Disponible en http://scielo.sld.cu/scielo.php?script=sci_arttext\&pid=S1729-519X2007000500004. Consultado $27 / 09 / 2018$

- (2008). La Bioética desde una óptica marxista. Educación Médica Superior, 22(3). Disponible en scielo.sld.cu/scielo.php?script=sci_arttext\&pid=S0864-21412008000300010. $\quad$ Consultado 25/11/2017.

Bravo Matarazzo, E. (1999). La ética del científico en Cuba. En J.R. Acosta, Bioética desde una perspectiva cubana. La Habana: Publicaciones Acuario.

Castro Ruz, F. (1991). Discurso pronunciado en la clausura del IV Congreso del Partido Comunista de Cuba. Disponible en http://www.cuba.cu/gobierno/discursos/1991/esp/f141091e.html. Consultado $25 / 11 / 2017$.

Cedeño Guerra, N. (2008). La responsabilidad penal del médico en Cuba. Su tratamiento teórico doctrinal y legislativo. Revista Jurídica. Disponible en http://dspace.uces.edu.ar:8180/xmlui/bitstream/handle/123456789/53/La_responsabilidad_pe nal_del_m\%C3\%A9dico.pdf?sequence=1. Consultado 22/11/2018.

Donoso Casanellas, J. (2000). Estudio sobre la Política Social cubana hasta el 2000. Disponible en http://www.rebelion.org/docs/16969.pdf. Consultado 27/11/2107.

Fleites González, G, J L. Soriano García y O. Torres Gemeil (1999). Comités de Ética Institucionales para la Investigación Clínica. En J.R. Acosta Sariego, Bioética. Desde una perspectiva cubana. La Habana: Publicaciones Acuario.

González, U; Grau, J.; Amarillo, M. A. (1997). La calidad de vida como un problema de la Bioética. En J. R. Acosta, Bioética. Desde una perspectiva cubana. La Habana: Publicaciones Acuario.

Hodelín Tablada, R. (2011). Bioética anglosajona en su 40 aniversario: el traspaso hacia América Latina y su llegada a Cuba. Disponible en http://bvs.sld.cu/revistas/san/vol_15_11_11/san201111.htm. Consultado 15/10/2018

Informe de la Dirección de Estudios Económicos del Banco Central de Cuba. "El Periodo Especial: la economía cubana en la década de los años 90". Disponible en http://www.bc.gov.cu/Anteriores/Otros/ecoomia\%20cubana.pdf. Consultado 27/11/2017

Milián Salaberri, E. (2009) Ley Torricelli, un puntal del bloqueo yanqui a Cub". Disponible en www.ain.cu/2009/octubre/23edtorricelli.htm. Consultado 14/10/2017.

Montt Momberg J. (1995) Bioética en América Latina y el Caribe. Santiago de Chile: Serie Documentos Programa Regional de Bioética OPS/OMS

Morales Domínguez, E. (1996). Economía y política del conflicto Cuba- EE.UU. en los años noventa. Economía y Desarrollo, 3(121).

Torres, R. (2001). Glosario de Bioética. La Habana: Publicaciones Acuario. 